\title{
Spectral Properties of a Fourth Order Differential Equation
}

\author{
Manfred Möller and Vyacheslav Pivovarchik
}

\begin{abstract}
The eigenvalue problem $y^{(4)}(\lambda, x)-\left(g y^{\prime}\right)^{\prime}(\lambda, x)=\lambda^{2} y(\lambda, x)$ with boundary conditions $y(\lambda, 0)=0, y^{\prime \prime}(\lambda, 0)=0, y(\lambda, a)=0, y^{\prime \prime}(\lambda, a)+i \alpha \lambda y^{\prime}(\lambda, a)=0$ is considered, where $g \in C^{1}[0, a]$ and $\alpha>0$. It is shown that the eigenvalues lie in the closed upper half-plane and on the negative imaginary axis. A formula for the asymptotic distribution of the eigenvalues is given and the location of the pure imaginary spectrum is investigated.
\end{abstract}

Keywords. Fourth-order differential equation, pure imaginary eigenvalues, eigenvalue distribution

Mathematics Subject Classification (2000). 34B07, 34L20

\section{Introduction}

Small transversal vibrations of a homogeneous beam compressed or stretched by a force $g$ can be described by the partial differential equation

$$
\frac{\partial^{4}}{\partial x^{4}} u(x, t)-\frac{\partial}{\partial x} g(x) \frac{\partial}{\partial x} u(x, t)=-\frac{\partial^{2}}{\partial t^{2}} u(x, t) .
$$

We suppose $g$ to be a sufficiently smooth real-valued function; throughout this paper $g \in C^{1}[0, a], a>0$, will be assumed. If $g>0$, then the beam is stretched, if $g<0$, then it is compressed. Let us impose the following boundary conditions at the left end

$$
u(0, t)=0,\left.\quad \frac{\partial^{2}}{\partial x^{2}} u(x, t)\right|_{x=0}=0,
$$

Manfred Möller: The John Knopfmacher Centre for Applicable Analysis and Number Theory, School of Mathematics, University of the Witwatersrand, WITS, 2050, South Africa; manfred@maths.wits.ac.za

VyacheslavPivovarchik: South-Ukrainian State Pedagogical University, Department of Applied Mathematics and Informatics, Staroportofrankovskaya str.26, 65091, Odessa, Ukraine; v.pivovarchik@paco.net 
which correspond to a hinge connection. Let the conditions at the right end be

$$
u(a, t)=0,\left.\quad \frac{\partial^{2}}{\partial x^{2}} u(x, t)\right|_{x=a}=-\left.\alpha \frac{\partial}{\partial t \partial x} u(x, t)\right|_{x=a} .
$$

The last boundary condition means that the hinge connected right end is subject to viscous friction $\alpha>0$ in the hinge. Substituting $u(x, t)=e^{i \lambda t} y(\lambda, x)$ we obtain the ordinary fourth oder differential equation

$$
y^{(4)}(\lambda, x)-\left(g y^{\prime}\right)^{\prime}(\lambda, x)=\lambda^{2} y(\lambda, x),
$$

with boundary conditions

$$
\begin{aligned}
y(\lambda, 0) & =0, \\
y^{\prime \prime}(\lambda, 0) & =0, \\
y(\lambda, a) & =0, \\
y^{\prime \prime}(\lambda, a)+i \alpha \lambda y^{\prime}(\lambda, a) & =0 .
\end{aligned}
$$

For technical reasons we also allow $\alpha$ to be zero in some of our statements.

It is shown that all eigenvalues $\lambda$ of the problem (1.1)-(1.5) lie in the closed upper half-plane or on the negative imaginary axis. A formula for the asymptotics of the eigenvalues is proved in Theorem 5.2, and the fine structure of the distribution of the pure imaginary eigenvalues is discussed in detail in Section 6 . For constant $g$ even more details about the pure imaginary eigenvalues can be given; this is done in Sections 7 and 8.

A similar result on the location of pure imaginary eigenvalues was obtained in [8] for the so-called generalized Regge problem, which is an analog of our problem generated by the Sturm-Liouville equation

$$
-y^{\prime \prime}+q(x) y=\lambda^{2} y, \quad y(0)=0, \quad y^{\prime}(a)+(i \alpha \lambda+\beta) y(a)=0 .
$$

It should be mentioned that the eigenvalue problem in [8] for a second order differential equation is simpler than the fourth order differential equations investigated in this paper. In particular, in [8], geometric multiplicities of pure imaginary eigenvalues are always one, there are no nonzero real eigenvalues, and the subsequence $\left\{\lambda_{k}^{(2)}\right\}$ described in Theorem 6.5 is absent.

Self-adjoint fourth order differential equations have been studied intensively, both for small transversal vibrations of beams and in hydrodynamics. We just mention a few of them. In [4] the study of inverse problems for the Timoshenko beam leads to a fourth order differential equation which is a quadratic pencil with respect to the eigenvalue parameter $b^{2}([4,(10)])$, with boundary conditions depending linearly on $b^{2}([4,(5),(7),(8)])$; it is shown that for certain choices of 
the parameters geometrically double eigenvalues exist. In [2] asymptotics of the eigenvalues of the corresponding boundary problems with different selfadjoint boundary conditions were investigated. The paper [10] is concerned with oscillations of solutions of the differential equation (without boundary conditions) and separation of their zeros. The problem of small transversal vibrations of the so-called Timoshenko beam with dissipative boundary conditions was considered in [9], where the asymptotics of eigenvalues were investigated. Finally, in [1] several higher order eigenvalue problems in hydrodynamics can be found.

\section{Birkhoff regularity}

For the definition of Birkhoff regularity we refer to [7, Definition 7.3.1].

Theorem 2.1. Replacing $\lambda$ with $\mu^{2}$, the eigenvalue problem (1.1)-(1.5) is Birkhoff regular for all $\alpha \geq 0$.

Proof. We first note that the characteristic function of (1.1) as defined in [7, (7.1.4)] is $\pi(\rho)=\rho^{4}-1$, and its zeros are $i^{k-1}, k=1, \ldots, 4$. Choosing

$$
C(x, \mu)=\operatorname{diag}\left(1, \mu, \mu^{2}, \mu^{3}\right)\left(i^{(k-1)(l-1)}\right)_{k, l=1}^{1}
$$

according to $[7$, Theorem 7.2.4.A], it follows that the boundary matrices defined in $[7,(7.3 .1)]$ are given by

$$
\begin{aligned}
W^{(0)}(\mu) & =\left(\begin{array}{llll}
1 & 0 & 0 & 0 \\
0 & 0 & 1 & 0 \\
0 & 0 & 0 & 0 \\
0 & 0 & 0 & 0
\end{array}\right) C(0, \mu)=\left(\begin{array}{cccc}
1 & 1 & 1 & 1 \\
\mu^{2} & -\mu^{2} & \mu^{2} & -\mu^{2} \\
0 & 0 & 0 & 0 \\
0 & 0 & 0 & 0
\end{array}\right) \\
W^{(1)}(\mu) & =\left(\begin{array}{ccccc}
0 & 0 & 0 & 0 \\
0 & 0 & 0 & 0 \\
1 & 0 & 0 & 0 \\
0 & i \alpha \mu^{2} & 1 & 0
\end{array}\right) C(a, \mu)=\left(\begin{array}{cccc}
0 & 0 & 0 & 0 \\
0 & 0 & 0 & 0 \\
1 & 1 & 1 & 1 \\
\gamma_{1} & \gamma_{2} & \gamma_{3} & \gamma_{4}
\end{array}\right),
\end{aligned}
$$

where $\gamma_{j}=i^{j} \alpha \mu^{3}+(-1)^{j-1} \mu^{2}, j=1, \ldots, 4$. Choosing $C_{2}(\mu)=\operatorname{diag}\left(1, \mu^{2}, 1, \mu^{3}\right)$ if $\alpha>0$, we obtain $C_{2}(\mu)^{-1} W^{(j)}(\mu)=W_{0}^{(j)}+O\left(\mu^{-1}\right)$, where

$$
W_{0}^{(0)}=\left(\begin{array}{cccc}
1 & 1 & 1 & 1 \\
1 & -1 & 1 & -1 \\
0 & 0 & 0 & 0 \\
0 & 0 & 0 & 0
\end{array}\right), \quad W_{0}^{(1)}=\left(\begin{array}{cccc}
0 & 0 & 0 & 0 \\
0 & 0 & 0 & 0 \\
1 & 1 & 1 & 1 \\
i \alpha & -\alpha & -i \alpha & \alpha
\end{array}\right) .
$$


According to [7, Definition 7.3.1 and Proposition 4.1.7], the matrices $\Delta$ of the problem are the four $4 \times 4$ diagonal matrices with 2 consecutive ones and 2 consecutive zeros in the diagonal in a cyclic arrangement. By [7, Definition 7.3.1] the problem (1.1)-(1.5) is Birkhoff regular if

$$
W_{0}^{(0)} \Delta+W_{0}^{(1)}(I-\Delta)
$$

is invertible for all four choices of $\Delta$. Now it is easy to see that, after a permutation of columns, the matrices (2.1) are block diagonal matrices consisting of $2 \times 2$ blocks taken from two consecutive columns (in the sense of cyclic arrangement) of the first two rows of $W_{0}^{(0)}$ and the last two rows of $W_{0}^{(1)}$, respectively. These matrices are obviously invertible. We thus have shown that the problem is Birkhoff regular.

If $\alpha=0$, then the same conclusion holds with $C_{2}(\mu)=\operatorname{diag}\left(1, \mu^{2}, 1, \mu^{2}\right)$.

\section{Differential operators and spectrum}

Let $A, K$ and $M$ be linear operators acting in $L_{2}(0, a) \oplus \mathbb{C}$ with domains

$$
\begin{aligned}
& D(A)=\left\{Y=\left(\begin{array}{c}
y(x) \\
y^{\prime}(a)
\end{array}\right): y \in W_{4}^{2}(0, a), y(0)=y^{\prime \prime}(0)=y(a)=0\right\} \\
& D(K)=D(M)=L_{2}(0, a) \oplus \mathbb{C},
\end{aligned}
$$

given by

$$
A Y=\left(\begin{array}{c}
y^{(4)}-\left(g y^{\prime}\right)^{\prime} \\
y^{\prime \prime}(a)
\end{array}\right), \quad K=\left(\begin{array}{ll}
0 & 0 \\
0 & 1
\end{array}\right), \quad M=\left(\begin{array}{ll}
I & 0 \\
0 & 0
\end{array}\right) .
$$

It is easy to check that $A=A^{*}$ is bounded below, $K \geq 0, M \geq 0, M+K=I$, and $\left.M\right|_{D(A)}>0$. Let us consider the operator pencil

$$
L(\lambda, \alpha)=\lambda^{2} M-i \alpha \lambda K-A
$$

with domain $D(L)=D(A)$ independent of the spectral parameter $\lambda$. Clearly, $L_{0}=L(0,0)=-A$ with $g=0$ is a Fredholm operator, and the operator $L(\lambda, \alpha)$ with general $g$ is a relatively compact perturbation of $L_{0}$. Therefore, by the perturbation theory of Fredholm operators, see [5, Theorem IV.5.26], $L(\lambda, \alpha)$ has a compact resolvent.

Similarly, according to $[7,(6.3 .1)]$, we can associate a boundary eigenvalue operator function $T(\lambda): W_{4}^{2}(0, a) \rightarrow L^{2}(0, a) \oplus \mathbb{R}^{4}$ given by

$$
T(\lambda) y=\left(\begin{array}{c}
y^{(4)}-\left(g y^{\prime}\right)^{\prime}-\lambda^{2} y \\
y(0) \\
y^{\prime \prime}(0) \\
y(a) \\
y^{\prime \prime}(a)+i \alpha \lambda y^{\prime}(a)
\end{array}\right)
$$


By Theorem 2.1, the operator function $T(\lambda)$ with $\lambda$ replaced by $\mu^{2}$ is Birkhoff regular. So the spectrum of $T(\lambda)$ consists of an infinite number of normal eigenvalues. Note that we write $T(\lambda)$ but $L(\lambda, \alpha)$ since we will mostly deal with the latter operator pencil and its dependence on $\alpha$ will frequently be made use of.

Since the components $y(0), y^{\prime \prime}(0), y(a)$ are independent of $\lambda$, it is easy to see that there is a one-to-one relation between a chain of an eigenvector and associated vectors $y_{0}, y_{1}, \ldots, y_{j}$ of $T(\lambda)$ and a chain of eigenvectors and associated vectors $Y_{0}, Y_{1}, \ldots, Y_{j}$ of $L(\lambda, \alpha)$. Therefore, when dealing with eigenvalues, their geometric and algebraic multiplicities, and chains of eigenvectors and associated vectors, we can take the operator pencils $L(\lambda, \alpha), T(\lambda)$ or, more informally, (1.1)-(1.5), whichever seems to be more appropriate. So we call the spectrum of the operator function $L(\lambda, \alpha)$ the spectrum of the problem (1.1)-(1.5).

The eigenvalues of (1.1)-(1.5) are continuous and piecewise analytic functions of the parameter $\alpha$ which can loose analyticity only at multiple eigenvalues, see, e.g., [3]. When $\alpha=0$ the eigenvalues are located on the real axis and on the imaginary axis symmetrically with respect to the real and to the imaginary axes. This is clear from the identity $L(\lambda, 0)=\lambda^{2} M-A$.

Lemma 3.1. All eigenvalues of $L(\lambda, \alpha), \alpha \geq 0$, lie in the closed upper half-plane and on the imaginary axis and are symmetric with respect to the imaginary axis.

Proof. Let $Y_{k}$ be an eigenvector corresponding to the eigenvalue $\lambda_{k}$ of the operator pencil $L(\lambda, \alpha)$. Then

$$
\lambda_{k}^{2}\left(M Y_{k}, Y_{k}\right)-i \lambda_{k} \alpha\left(K Y_{k}, Y_{k}\right)-\left(A Y_{k}, Y_{k}\right)=0
$$

Taking the imaginary part of this equation we obtain

$$
2 \operatorname{Re} \lambda_{k} \operatorname{Im} \lambda_{k}\left(M Y_{k}, Y_{k}\right)-\alpha \operatorname{Re} \lambda_{k}\left(K Y_{k}, Y_{k}\right)=0 .
$$

This yields either $\operatorname{Re} \lambda_{k}=0$ or

$$
2 \operatorname{Im} \lambda_{k}\left(M Y_{k}, Y_{k}\right)-\alpha\left(K Y_{k}, Y_{k}\right)=0 .
$$

Since $\left.M\right|_{D(A)}>0$ and $K \geq 0$, we obtain $\operatorname{Im} \lambda_{k} \geq 0$. The symmetry follows from

$$
(-\bar{\lambda})^{2} M \bar{Y}-i \alpha(-\bar{\lambda}) K \bar{Y}-A \bar{Y}=\overline{\lambda^{2} M Y-i \alpha \lambda K Y-A Y}
$$

for all $\lambda \in \mathbb{C}$ and $Y \in D(L)$, where $\bar{Y}$ denotes the conjugate complex of $Y$.

Lemma 3.2. All nonzero real eigenvalues of $L(\lambda, \alpha), \alpha>0$, (if any) are semisimple, i.e., the corresponding eigenvectors do not possess associated vectors. All real eigenvalues of $L(\lambda, \alpha), \alpha>0$, are independent of $\alpha$. 
Proof. Let $\lambda_{0}$ be a real nonzero eigenvalue, $Y_{0}$ a corresponding eigenvector and assume that there is a corresponding associated vector $Y_{1}$. Then

$$
\begin{aligned}
\lambda_{0}^{2} M Y_{0}-i \lambda_{0} \alpha K Y_{0}-A Y_{0} & =0 \\
2 \lambda_{0} M Y_{0}-i \alpha K Y_{0}+\lambda_{0}^{2} M Y_{1}-i \lambda_{0} \alpha K Y_{1}-A Y_{1} & =0 .
\end{aligned}
$$

From (3.1) we obtain

$$
\lambda_{0}^{2}\left(M Y_{0}, Y_{0}\right)-i \lambda_{0} \alpha\left(K Y_{0}, Y_{0}\right)-\left(A Y_{0}, Y_{0}\right)=0
$$

which shows

$$
\left(K Y_{0}, Y_{0}\right)=0 .
$$

Due to $K \geq 0$ equation (3.3) implies

$$
K Y_{0}=0 .
$$

Then from (3.1) we deduce

$$
\lambda_{0}^{2} M Y_{0}-A Y_{0}=0
$$

and (3.2) leads to

$$
2 \lambda_{0}\left(M Y_{0}, Y_{0}\right)-i \alpha\left(K Y_{0}, Y_{0}\right)+\lambda_{0}^{2}\left(M Y_{1}, Y_{0}\right)-i \lambda_{0} \alpha\left(K Y_{1}, Y_{0}\right)-\left(A Y_{1}, Y_{0}\right)=0 .
$$

Using (3.4) this gives

$$
2 \lambda_{0}\left(M Y_{0}, Y_{0}\right)+\left(Y_{1}, \lambda_{0}^{2} M Y_{0}\right)-\left(Y_{1}, A Y_{0}\right)=0,
$$

and then (3.5) implies $\left(M Y_{0}, Y_{0}\right)=0$, which contradicts $\left.M\right|_{D(A)}>0$.

Note that (3.5) is also true for $\lambda_{0}=0$ if (3.1) is satisfied, whence it follows that any real eigenvalue $\lambda_{0}$ is independent of $\alpha$.

Lemma 3.3. Let $\lambda=-i \tau, \tau>0$, be an eigenvalue of $L(\lambda, \alpha), \alpha \geq 0$. Then $\lambda$ is semisimple.

Proof. Let $Y_{0}$ be an eigenvector for the eigenvalue $-i \tau$ and suppose there exists a corresponding associated vector $Y_{1}$. Then

$$
\begin{array}{r}
-\tau^{2} M Y_{0}-\tau \alpha K Y_{0}-A Y_{0}=0 \\
-2 i \tau M Y_{0}-i \alpha K Y_{0}-\tau^{2} M Y_{1}-\tau \alpha K Y_{1}-A Y_{1}=0 .
\end{array}
$$

Applying (3.8) to $Y_{0}$ we obtain

$$
-2 i \tau\left(M Y_{0}, Y_{0}\right)-i \alpha\left(K Y_{0}, Y_{0}\right)-\tau^{2}\left(M Y_{1}, Y_{0}\right)-\tau \alpha\left(K Y_{1}, Y_{0}\right)-\left(A Y_{1}, Y_{0}\right)=0
$$

or, what is the same,

$$
-2 i \tau\left(M Y_{0}, Y_{0}\right)-i \alpha\left(K Y_{0}, Y_{0}\right)+\left(Y_{1},-\tau^{2} M Y_{0}-\tau \alpha K Y_{0}-A Y_{0}\right)=0 .
$$

Due to (3.7) this implies $2 \tau\left(M Y_{0}, Y_{0}\right)+\alpha\left(K Y_{0}, Y_{0}\right)=0$ which contradicts the inequalities $\tau>0,\left.M\right|_{D(A)}>0, \alpha \geq 0$, and $K \geq 0$. 
Lemma 3.4. Let $\lambda_{k}(\alpha)=i \tau, \tau \in \mathbb{R} \backslash\{0\}$, be an eigenvalue of $L(\lambda, \alpha), \alpha \geq 0$. Then:

1. $\operatorname{Re} \dot{\lambda}_{k}(0)=0$ and $\operatorname{Im} \dot{\lambda}_{k}(0) \geq 0$; here ${ }^{\cdot}$ means derivative with respect to $\alpha$.

2. If $\tau<0$, then $\operatorname{Re} \dot{\lambda}_{k}(\alpha)=0$ and $\operatorname{Im} \dot{\lambda}_{k}(\alpha) \geq 0$ for all $\alpha \geq 0$.

3. If 0 is an eigenvalue of $L(\lambda, \alpha)$ for some $\alpha \geq 0$, then it is an eigenvalue for all $\alpha \geq 0$, its geometric multiplicity is the same for all $\alpha \geq 0$, whereas its algebraic multiplicity is the same for all $\alpha>0$. If the geometric multiplicity of the eigenvalue 0 is 1 , then the algebraic multiplicity for $\alpha=0$ is 2 , whereas its algebraic multiplicity for $\alpha>0$ is 1 or 2 . If the geometric multiplicity of the eigenvalue 0 is 2 , then the algebraic multiplicity for $\alpha=0$ is 4, whereas its algebraic multiplicity for $\alpha>0$ is 2 or 3 .

Proof. First we have to justify the differentiability at $\alpha_{0}=0$ for all $\lambda=i \tau$, $\tau \neq 0$, and for $\alpha_{0}>0$ and $\lambda=i \tau, \tau<0$. This is clear if the eigenvalue is simple. Since the eigenvalues under consideration are semisimple by Lemma 3.3 - for $\alpha=0$ and $\tau>0$ observe the symmetry of the problem - nonsimple eigenvalues have geometric and algebraic multiplicity 2, where we have used that due to the boundary conditions (1.2), (1.3), the geometric multiplicity can be at most 2 . Then it follows from general results, see, e.g., [3] or [6], that the eigenvalues are differentiable. But in our case this is easily seen. Indeed, since there is an eigenvector $y$ of (1.1)-(1.5) with $y^{\prime}(a)=0$ at $\lambda_{k}\left(\alpha_{0}\right)$, one eigenvalue $\lambda_{0}=\lambda_{k}\left(\alpha_{0}\right)$ is an eigenvalue for all $\alpha$. If now $m(\lambda, \alpha)$ denotes the characteristic determinant of (1.1)-(1.5), which depends analytically on $\lambda$ and $\alpha$, then also

$$
\tilde{m}(\lambda, \alpha)=\frac{m(\lambda, \alpha)}{\lambda-\lambda_{0}}
$$

depends analytically on $\lambda$ and $\alpha$. But now $\lambda_{0}$ is a simple zero of $\tilde{m}(\lambda, \alpha)$ at $\lambda_{0}$, and thus the other eigenvalue $\lambda_{k^{\prime}}(\alpha)$ depends analytically on $\alpha$ as well.

If $\lambda_{k}=i \tau, \tau \in \mathbb{R}$, is an eigenvalue of $L(\lambda, \alpha), \alpha \geq 0$, then

$$
-\tau^{2}(M Y, Y)+\tau \alpha(K Y, Y)-(A Y, Y)=0 .
$$

Here $Y$ is an eigenvector corresponding to $\lambda_{k}$ which depends analytically on $\alpha$. Differentiating (3.9) with respect to $\alpha$ we obtain

$$
\begin{aligned}
& -\tau^{2}(M Y, \dot{Y})+\tau \alpha(K Y, \dot{Y})-(A Y, \dot{Y})-\tau^{2}(M \dot{Y}, Y)+\tau \alpha(K \dot{Y}, Y) \\
& -(A \dot{Y}, Y)+2 i \tau \dot{\lambda_{k}}(M Y, Y)-i \alpha \dot{\lambda_{k}}(K Y, Y)+\tau(K Y, Y)=0 .
\end{aligned}
$$

Obviously,

$$
\begin{aligned}
-\tau^{2}(M Y, \dot{Y})+\tau \alpha(K Y, \dot{Y})-(A Y, \dot{Y}) & =\left(\left(-\tau^{2} M+\tau \alpha K-A\right) Y, \dot{Y}\right)=0 \\
-\tau^{2}(M \dot{Y}, Y)+\tau \alpha(K \dot{Y}, Y)-(A \dot{Y}, Y) & =\left(\dot{Y},\left(-\tau^{2} M+\tau \alpha K-A\right) Y\right)=0
\end{aligned}
$$


Substituting these equations into (3.10) we obtain

$$
\dot{\lambda_{k}}=\frac{i \tau(K Y, Y)}{2 \tau(M Y, Y)-\alpha(K Y, Y)},
$$

provided the denominator is different from zero. Since $\left.M\right|_{D(A)}>0$ and $K \geq 0$, statements 1 and 2 follow.

We turn our attention to statement 3. Since $L(0, \alpha)=L(0,0)$, the statement about the geometric multiplicity is obvious. Also, since $L(\lambda, 0)$ is a function of $\lambda^{2}$, each eigenvector of $L(\lambda, 0)$ corresponding to the eigenvalue 0 has an associated vector zero. Assume there is an eigenvector $Y_{0}$ corresponding to the eigenvalue 0 of $L(\lambda, 0)$ which has a chain of associated vectors $Y_{1}, Y_{2}$, i. e.,

$$
-A Y_{0}=0, \quad-A Y_{1}=0, \quad M Y_{0}-A Y_{2}=0 .
$$

Taking the scalar product with $Y_{0}$ in the last equation and observing the first equation and the self-adjointness of $A$, we infer

$$
0=\left(M Y_{0}, Y_{0}\right)-\left(A Y_{2}, Y_{0}\right)=\left(M Y_{0}, Y_{0}\right),
$$

which gives $Y_{0}=0$ since $\left.M\right|_{D(A)}>0$; a contradiction as $Y_{0}$ is an eigenvector. The assertions for $\alpha=0$ are proved.

Now let $\alpha>0$. If 0 is an eigenvalue of $L(\lambda, \alpha)$ with an eigenvector $Y_{0}$ which has an associated vector $Y_{1}$, then

$$
-A Y_{0}=0, \quad-i \alpha K Y_{0}-A Y_{1}=0 .
$$

It follows that

$$
0=-i \alpha\left(K Y_{0}, Y_{0}\right)-\left(A Y_{1}, Y_{0}\right)=-i \alpha\left(K Y_{0}, Y_{0}\right)
$$

and $K \geq 0$ implies

$$
K Y_{0}=0,
$$

and therefore $y_{0}^{\prime}(a)=0$. This has two consequences: firstly, the solution $y_{0}$ of (1.1)-(1.5) is independent of $\alpha$, and secondly, the boundary conditions at $a$ are $y_{0}(a)=y_{0}^{\prime}(a)=y_{0}^{\prime \prime}(a)=0$, so that at most one linearly independent eigenvector can have an associated vector. Assume this eigenvector $Y_{0}$ has a chain $Y_{1}, Y_{2}$ of associated vectors, i. e., (3.12) and

$$
M Y_{0}-i \alpha K Y_{1}-A Y_{2}=0
$$

hold. This leads to

$0=\left(M Y_{0}, Y_{0}\right)-i \alpha\left(K Y_{1}, Y_{0}\right)-\left(A Y_{2}, Y_{0}\right)=\left(M Y_{0}, Y_{0}\right)-i \alpha\left(Y_{1}, K Y_{0}\right)-\left(Y_{2}, A Y_{0}\right)$.

By (3.12) and (3.13) we thus arrive at the contradiction $\left(M Y_{0}, Y_{0}\right)=0$. Altogether, the algebraic multiplicity is at most 3 and independent of $\alpha$. This completes the proof of the assertions for $\alpha>0$. 


\section{Asymptotics of eigenvalues for $g=0$}

In this section we consider the eigenvalue problem (1.1)-(1.5) with $g=0$. A formula for the asymptotic distribution of eigenvalues is proved, which will be used to obtain the corresponding formula for general $\mathrm{g}$. Since we also want to count all eigenvalues with their proper multiplicities, we have to carefully define the corresponding characteristic equation. So we take the canonical fundamental system $y_{j}, j=1, \ldots, 4$, with $y_{j}^{(m)}(0)=\delta_{j, m+1}$ for $m=0, \ldots, 3$, which is analytic on $\mathbb{C}$ with respect to $\lambda$. Because of the boundary conditions $y(0)=y^{\prime \prime}(0)=0$ we only need $y_{2}$ and $y_{4}$. We put $\mu=\sqrt{\lambda}, \lambda \neq 0$. It is easy to see that

$$
\begin{aligned}
& y_{2}(x)=\frac{1}{2 \mu} \sin (\mu x)+\frac{1}{2 \mu} \sinh (\mu x) \\
& y_{4}(x)=-\frac{1}{2 \mu^{3}} \sin (\mu x)+\frac{1}{2 \mu^{3}} \sinh (\mu x),
\end{aligned}
$$

with the proper interpretation as a limit for $\lambda=0$. Representing the boundary conditions (1.4), (1.5) by functionals $B_{1}, B_{2}$, the (reduced) characteristic matrix of the boundary value problem, as represented by $T(\lambda)$ defined in $\S 3$, becomes

$$
M=\left(\begin{array}{l}
B_{1} \\
B_{2}
\end{array}\right)\left(\begin{array}{ll}
y_{2} & y_{4}
\end{array}\right) .
$$

Since

$$
\left(\begin{array}{ll}
y_{2}(x) & y_{4}(x)
\end{array}\right)=\left(\begin{array}{ll}
\sin (\mu x) & \sinh (\mu x)
\end{array}\right)\left(\begin{array}{cc}
\frac{1}{2 \mu} & -\frac{1}{2 \mu^{3}} \\
\frac{1}{2 \mu} & \frac{1}{2 \mu^{3}}
\end{array}\right),
$$

it is easy to see that the characteristic equation $2 \operatorname{det} M=0$ becomes

$$
\varphi(\mu):=i \alpha \varphi_{0}(\mu)+\varphi_{1}(\mu)=0,
$$

where

$$
\begin{aligned}
& \varphi_{0}(\mu)=\frac{1}{\mu}(\sin (\mu a) \cosh (\mu a)-\sinh (\mu a) \cos (\mu a)) \\
& \varphi_{1}(\mu)=\frac{2}{\mu^{2}} \sin (\mu a) \sinh (\mu a) .
\end{aligned}
$$

First we will give an asymptotic distribution with exact indexing for the zeros of $\varphi_{0}$. Obviously, $\frac{1}{\mu^{2}} \varphi_{0}(\mu)$ is the characteristic function of the above problem with boundary condition (1.5) replaced by $y^{\prime}(a)=0$. This problem has an operator realization $B-\lambda^{2} I$ with a non-negative selfadjoint operator $B$, so that the zeros of $\varphi_{0}$, as a function of $\lambda=\mu^{2}$, are real. Hence the zeros of $\varphi_{0}$ lie on the real and imaginary axes.

A power series expansion shows that 0 is a double eigenvalue, denoted by $\tilde{\mu}_{0}^{ \pm}$. Next observe that $\varphi_{0}(\mu)=0$ implies $\cos (\mu a) \neq 0$ and $\cosh (\mu a) \neq 0$, whence the 
nonzero zeros of $\varphi_{0}$ are given by those $\mu \neq 0$ for which $\tan (\mu a)=\tanh (\mu a)$. Since $\tan ^{\prime}(x) \geq 1$ and $\tanh ^{\prime}(x)<1$ for $x \in \mathbb{R} \backslash\{0\}$, the function $\tan (x)-\tanh (x)$ is increasing with positive derivative on each interval $\left(\left(k-\frac{1}{2}\right) \pi,\left(k+\frac{1}{2}\right) \pi\right)$, with the exception of the point 0 . On each of these intervals, the function moves from $-\infty$ to $+\infty$, so that we have exactly one simple zero $\tilde{\mu}_{k}^{ \pm}$of $\tan (\mu a)-\tanh (\mu a)$ in each interval $\left(\left( \pm k-\frac{1}{2}\right) \frac{\pi}{a},\left( \pm k+\frac{1}{2}\right) \frac{\pi}{a}\right), k$ a positive integer, and no nonzero zero in $\left(-\frac{\pi}{2 a}, \frac{\pi}{2 a}\right)$. Since $\tanh x \rightarrow 1$ as $x \rightarrow \infty$ and $\mu_{k}^{-}=-\mu_{k}^{+}$, we thus have

$$
\tilde{\mu}_{k}^{+}=k \frac{\pi}{a}+\frac{\pi}{4 a}+o\left(k^{-1}\right), \quad \tilde{\mu}_{k}^{-}=-k \frac{\pi}{a}-\frac{\pi}{4 a}+o\left(k^{-1}\right), \quad k=1,2, \ldots
$$

As $\tan (i \gamma a)=i \tanh (\gamma a)$ and $\tanh (i \gamma a)=i \tan (\gamma a)$, the nonzero pure imaginary zeros of $\varphi_{0}$ are simple and of the form

$$
\tilde{\mu}_{-k}^{+}=i k \frac{\pi}{a}+i \frac{\pi}{4 a}+o\left(k^{-1}\right), \quad \tilde{\mu}_{-k}^{-}=-i k \frac{\pi}{a}-i \frac{\pi}{4 a}+o\left(k^{-1}\right), \quad k=1,2, \ldots,
$$

which shows that the zeros of $\varphi_{0}$, counted with multiplicity, are $\tilde{\mu}_{k}^{ \pm}, k \in \mathbb{Z}$.

Let $\varphi_{2}(\mu)=\operatorname{coth}(\mu a)-\cot (\mu a)$. Since

$$
\cot \left(\left(\frac{j \pi}{a}+i \gamma\right) a\right)=\cot (i \gamma a)=i \operatorname{coth}(\gamma) \in i \mathbb{R} \cup\{\infty\}
$$

for $j \in \mathbb{Z}, \gamma \in \mathbb{R}$, we have

$$
|\cot (\mu a) \pm 1| \geq 1 \quad \text { for } \mu=\frac{j \pi}{a}+i \gamma, \gamma \in \mathbb{R} .
$$

For $\mu a=x+i y$ we conclude

$$
\operatorname{coth}(\mu a)=\frac{e^{x+i y}+e^{-x-i y}}{e^{x+i y}-e^{-x-i y}} \rightarrow \pm 1
$$

uniformly in $y$ as $x \rightarrow \pm \infty$. Hence there is $j_{0} \in \mathbb{N}$ such that

$$
\left|\operatorname{coth}\left(\left(\frac{j \pi}{a}+i \gamma\right) a\right)-(-1)^{\operatorname{sgn}(j)}\right|<\frac{1}{2}
$$

for all $j \in \mathbb{Z}$ with $|j| \geq j_{0}$ and $\gamma \in \mathbb{R}$. These two estimates lead to

$$
\left|\varphi_{2}(\mu)\right| \geq \frac{1}{2} \quad \text { for } \mu=\frac{j \pi}{a}+i \gamma, j \in \mathbb{Z},|j| \geq j_{0}, \gamma \in \mathbb{R} .
$$

By interchanging coth and cot we obtain the same estimate for $\mu=\gamma+i \frac{j \pi}{a}$, $j \in \mathbb{Z},|j| \geq j_{0}, \gamma \in \mathbb{R}$. Hence, for $\mu$ on the square with vertices $\pm j \frac{\pi}{a} \pm i j \frac{\pi}{a}$, where $j \in \mathbb{N},|j| \geq \max \left\{j_{0}, \frac{5 a}{\pi \alpha}\right\}$,

$$
\begin{aligned}
\alpha\left|\varphi_{0}(\mu)\right| & =\frac{\alpha}{\mu}\left|\varphi_{2}(\mu)\right||\sin (\mu a) \sinh (\mu a)| \\
& \geq \frac{\alpha}{2|\mu|}|\sin (\mu a) \sinh (\mu a)| \\
& =\frac{\alpha|\mu|}{4}\left|\varphi_{1}(\mu)\right| \\
& >\left|\varphi_{1}(\mu)\right| .
\end{aligned}
$$


Hence, by Rouché's theorem, $\varphi$ and $\varphi_{0}$ have the same number of zeros inside the square. Putting

$$
\begin{aligned}
& \varphi_{00}(\mu)=\sin (\mu a)-\cos (\mu a), \\
& \varphi_{01}(\mu)=(1-\tanh (\mu a)) \cos (\mu a)-\frac{2 i}{\mu \alpha} \sin (\mu a) \tanh (\mu a),
\end{aligned}
$$

we obtain

$$
\varphi_{02}(\mu):=\frac{\mu \varphi(\mu)}{i \alpha \cosh (\mu a)}=\varphi_{00}(\mu)+\varphi_{01}(\mu)
$$

Note that

$$
\mu_{k}^{00}=k \frac{\pi}{a}+\frac{\pi}{4 a}, \quad k \in \mathbb{Z},
$$

are the zeros of $\varphi_{00}$. Let $C_{k}^{\rho}$ be the circle of radius $\rho<\frac{\pi}{2 a}$ with centre at $\mu_{k}^{00}$. Due to $\rho<\frac{\pi}{2 a}$ theses circles do not intersect. Since $\left|\varphi_{00}\right|$ is periodic with period $\frac{\pi}{a}$, there is a constant $p(\rho)>0$ such that $\left|\varphi_{00}(\mu)\right|>p(\rho)$ for all $\mu \in C_{k}^{\rho}$ and all $k \in \mathbb{Z}$. We estimate $\varphi_{01}$ on these circles for sufficiently large positive $k$ :

$$
\begin{aligned}
& |1-\tanh (\mu a)|=\frac{2}{\left|e^{2 \mu a}+1\right|}<\frac{2}{e^{2 \operatorname{Re} \mu a}-1}<C_{1} e^{-\operatorname{Re} \mu a} \\
& |\cos (\mu a)|<C_{2}, \quad|\sin (\mu a)|<C_{2}, \quad|\tanh (\mu a)|<C_{3}
\end{aligned}
$$

for $\mu \in C_{k}^{\rho}$, where the constants $C_{j}$ are independent of $\rho$ and $k$ is large enough. Thus we obtain

$$
\left|\varphi_{01}(\mu)\right|<\frac{C_{4}}{|\mu|}+C_{5} e^{-\operatorname{Re} \mu a}
$$

for $\mu \in C_{k}^{\rho}$ and $k \geq k_{0}(\rho)$ large enough. Since the right hand side tends to 0 as $\operatorname{Re} \mu \rightarrow \infty$, it follows that

$$
\left|\varphi_{01}(\mu)\right|<\left|\varphi_{00}(\mu)\right|, \quad\left(\mu \in C_{k}^{\rho}, k>k_{0}(\rho)\right) .
$$

Applying Rouché's theorem we obtain that each of the circles (for $k$ large enough) contains exactly one zero of $\varphi_{02}$, and thus exactly one zero of $\varphi$.

Using that $\varphi$ is an even function and the symmetry argument of Lemma 3.1, there are corresponding sequence of zeros of $\varphi$ on the negative real axis and along the imaginary axis.

As we have shown earlier, inside sufficently large squares, $\varphi$ and $\varphi_{0}$ have the same number of zeros, whereas the last result shows that there are zeros which have the same asymptotics as the zeros of $\varphi_{0}$. We summarize the results of this section, taking Lemma 3.1 into account: 
Lemma 4.1. For $g=0$, there is a positive integer $k_{0}$ such that the eigenvalues $\hat{\lambda}_{k}, k \in \mathbb{Z}$, of the problem (1.1)-(1.5), counted with multiplicity, can be enumerated in such a way that the eigenvalues $\hat{\lambda}_{k}$ are pure imaginary for $|k|<k_{0}$, and $\hat{\lambda}_{-k}=-\hat{\lambda}_{k}$ for $|k| \geq k_{0}$, where $\hat{\lambda}_{k}=\left(\hat{\mu}_{k}^{ \pm}\right)^{2}$ with

$$
\begin{array}{ll}
\hat{\mu}_{k}^{ \pm}= \pm\left(k \frac{\pi}{a}+\frac{\pi}{4 a}\right)+o(1), & \text { if } k>0 \\
\hat{\mu}_{k}^{ \pm}= \pm i\left(k \frac{\pi}{a}+\frac{\pi}{4 a}\right)+o(1), & \text { if } k<0 .
\end{array}
$$

In particular, there is an odd number of pure imaginary eigenvalues.

\section{Asymptotics of eigenvalues}

We again replace $\lambda$ with $\mu^{2}$. Then, according to [7, Theorem 8.2.1], (1.1) has an asymptotic fundamental system $\left\{\eta_{1}, \eta_{2}, \eta_{3}, \eta_{4}\right\}$ of the form

$$
\eta_{\nu}^{(j)}(x, \mu)=\delta_{\nu, j}(x, \mu) e^{\mu i^{\nu-1} x}
$$

where

$$
\delta_{\nu, j}(x, \mu)=\frac{d^{j}}{d x^{j}}\left\{\sum_{r=0}^{k}\left(\mu i^{\nu-1}\right)^{-r} \varphi_{r}(x) e^{\mu i^{\nu-1} x}\right\} e^{-\mu i^{\nu-1} x}+o\left(\mu^{-k+j}\right),
$$

$k=0,1, \ldots$, and $k$ can be chosen to be sufficiently large if $g$ is sufficiently regular. Since the coefficient of $y^{(3)}$ in (1.1) is zero, [7, (8.2.3)] gives $\varphi_{0}(x)=1$. Further functions $\varphi_{1}, \varphi_{2}, \ldots$ will be determined when the need arises.

It now follows that the characteristic function of (1.1)-(1.5) is

$$
D(\mu)=\operatorname{det}\left(\gamma_{j, k} \exp \left(\varepsilon_{j, k}\right)\right)_{j, k=1}^{4}
$$

where

$$
\begin{array}{ll}
\gamma_{1, k}=\delta_{k, 0}(0, \mu), & \gamma_{2, k}=\delta_{k, 2}(0, \mu) \\
\gamma_{3, k}=\delta_{k, 0}(a, \mu), & \gamma_{4, k}=\delta_{k, 2}(a, \mu)+i \alpha \mu^{2} \delta_{k, 1}(a, \mu) \\
\varepsilon_{1, k}=\varepsilon_{2, k}=0, & \varepsilon_{3, k}=\varepsilon_{4, k}=i^{k-1} \mu a .
\end{array}
$$

For $g=0$ we already know the asymptotic distribution of the eigenvalues, see Lemma 4.1. Denote the corresponding function $D$ by $D_{0}$. Due to the Birkhoff regularity, $g$ only influences lower order terms in $D$, and therefore it follows from the estimates in [7, Appendix A.2] that, away from small disks around the zeros of $D_{0},\left|D(\lambda)-D_{0}(\lambda)\right|<\left|D_{0}(\lambda)\right|$ if $|\lambda|$ is sufficiently large. The function $D(\lambda)$ is not analytic, but this estimate extends to the analytic equivalents with, e. g., 
a fundamental system $y_{j}, j=1, \ldots, 4$, with $y_{j}^{(m)}(0)=\delta_{j, m+1}$ for $m=0, \ldots, 3$, since these fundamental systems for general $g$ and $g=0$ are asymptotically close. Hence applying Rouché's theorem both to large circles centred at zero avoiding the small disks and to the boundaries of the small discs which are sufficiently far away from 0 , it follows that the eigenvalue problem for general $g$ has the same asymptotic distribution as for $g=0$. Hence Lemma 4.1 leads to

Lemma 5.1. For $g \in C^{1}[0, a]$ there is a positive integer $k_{0}$ such that the eigenvalues $\lambda_{k}, k \in \mathbb{Z}$, of the problem (1.1)-(1.5), counted with multiplicity, can be enumerated in such a way that the eigenvalues $\lambda_{k}$ are pure imaginary for $|k|<k_{0}, \lambda_{-k}=-\overline{\lambda_{k}}$ for $k \geq k_{0}$, where $\lambda_{k}=\mu_{k}^{2}$ with

$$
\mu_{k}=k \frac{\pi}{a}+\frac{\pi}{4 a}+o(1)
$$

as $k \rightarrow \infty$. In particular, there is an odd number of pure imaginary eigenvalues.

In [7, Appendix A] an estimate below of $D(\mu)$ has been given. With the methods used there one can also obtain more precise estimates of the location of the zeros of $D$. To this end we first observe that $D$ has the form

$$
D(\mu)=\sum_{m=1}^{5} \psi_{m}(\mu) e^{\omega_{m} \mu a}
$$

where the $\psi_{j}(\mu)$ are polynomials in $\delta_{\nu, k}(0, \mu)$ and $\delta_{\nu, k}(a, \mu)$ and $\omega_{1}=1+i$, $\omega_{2}=-1+i, \omega_{3}=-1-i, \omega_{4}=1-i, \omega_{5}=0$. For example, we can write

$$
D_{1}(\mu):=D(\mu) e^{-\omega_{1} \mu a}=\psi_{1}(\mu)+\sum_{m=2}^{5} \psi_{m}(\mu) e^{\left(\omega_{m}-\omega_{1}\right) \mu a} .
$$

It now follows from $\omega_{2}-\omega_{1}=-2, \omega_{3}-\omega_{1}=-2-2 i, \omega_{4}-\omega_{1}=-2 i, \omega_{5}-\omega_{1}=$ $-1-i$, that for $\arg \mu \in\left[-\frac{3 \pi}{8}, \frac{\pi}{8}\right]$ we have $\left|e^{\left(\omega_{m}-\omega_{1}\right) \mu a}\right| \leq e^{-\sin \frac{\pi}{8}|\mu| a}$ for $\mu=2,3,5$, and these terms therefore can be absorbed by $\psi_{1}(\mu)$ as they are of the form $o\left(\mu^{-s}\right)$ for any integer $s$. Hence, in the sector $\arg \mu \in\left[-\frac{3 \pi}{8}, \frac{\pi}{8}\right]$,

$$
D_{1}(\mu)=\psi_{1}(\mu)+\psi_{4}(\mu) e^{\left(\omega_{4}-\omega_{1}\right) \mu a}=\psi_{1}(\mu)+\psi_{4}(\mu) e^{-2 i \mu a} .
$$

To find the eigenvalue asymptotics along the positive real axis observe that the Birkhoff regularity, see Theorem 2.1, implies that $\psi_{1}$ and $\psi_{2}$ have nonzero terms apart from the $o$-terms. Due to the symmetry of the problem, the asymptotics on the other half-axes are not explicitly needed.

We now want to find the asymptotics (note that $\tau_{k, 0}=\frac{\pi}{4 a}$ )

$$
\mu_{k}=k \frac{\pi}{a}+\tau_{k}, \quad \tau_{k}=\sum_{m=0}^{n} \tau_{k, m} k^{-m}+o\left(k^{-n}\right), \quad k=1,2, \ldots
$$


Next we find the term $\tau_{k, 1}$ in the expansion of $\tau_{k}$ in (5.4). We calculate

$$
\begin{aligned}
\psi_{1}(\mu)= & \left|\begin{array}{ll}
\gamma_{1,3} & \gamma_{1,4} \\
\gamma_{2,3} & \gamma_{2,4}
\end{array}\right| \cdot\left|\begin{array}{ll}
\gamma_{3,1} & \gamma_{3,2} \\
\gamma_{4,1} & \gamma_{4,2}
\end{array}\right| \\
= & {\left[\delta_{3,0}(0, \mu) \delta_{4,2}(0, \mu)-\delta_{4,0}(0, \mu) \delta_{3,2}(0, \mu)\right] } \\
& \times\left\{\delta_{1,0}(a, \mu)\left[\delta_{2,2}(a, \mu)+i \alpha \mu^{2} \delta_{2,1}(a, \mu)\right]\right. \\
& \left.-\delta_{2,0}(a, \mu)\left[\delta_{1,2}(a, \mu)+i \alpha \mu^{2} \delta_{1,1}(a, \mu)\right]\right\} \\
\psi_{4}(\mu)= & \left|\begin{array}{ll}
\gamma_{1,2} & \gamma_{1,3} \\
\gamma_{2,2} & \gamma_{2,3}
\end{array}\right| \cdot\left|\begin{array}{ll}
\gamma_{3,1} & \gamma_{3,4} \\
\gamma_{4,1} & \gamma_{4,4}
\end{array}\right| \\
= & {\left[\delta_{2,0}(0, \mu) \delta_{3,2}(0, \mu)-\delta_{3,0}(0, \mu) \delta_{2,2}(0, \mu)\right] } \\
& \times\left\{\delta_{1,0}(a, \mu)\left[\delta_{4,2}(a, \mu)+i \alpha \mu^{2} \delta_{4,1}(a, \mu)\right]\right. \\
& \left.-\delta_{4,0}(a, \mu)\left[\delta_{1,2}(a, \mu)+i \alpha \mu^{2} \delta_{1,1}(a, \mu)\right]\right\} .
\end{aligned}
$$

Then $\psi_{1}(\mu)=2 \alpha(1+i) \mu^{5}+O\left(\mu^{4}\right), \psi_{4}(\mu)=2 \alpha(1-i) \mu^{5}+O\left(\mu^{4}\right)$ as $\mu \rightarrow \infty$,

$$
\begin{aligned}
& \mu^{-5} \psi_{1}(\mu)=2 \alpha(1+i)+\psi_{1,1} \mu^{-1}+o\left(\mu^{-1}\right) \\
& \mu^{-5} \psi_{4}(\mu)=2 \alpha(1-i)+\psi_{4,1} \mu^{-1}+o\left(\mu^{-1}\right) .
\end{aligned}
$$

Also note that

$$
\frac{1}{\mu_{k}}=\left(k \frac{\pi}{a}+\frac{\pi}{4 a}+o(1)\right)^{-1}=\frac{a}{\pi k}\left(1-\frac{1}{4 k}\right)+o\left(k^{-2}\right)
$$

implies

$$
\begin{gathered}
\mu_{k}^{-5} \psi_{1}\left(\mu_{k}\right)=2 \alpha(1+i)+\frac{1}{k} \frac{\psi_{1,1} a}{\pi}+o\left(k^{-1}\right) \\
\mu_{k}^{-5} \psi_{4}\left(\mu_{k}\right)=2 \alpha(1-i)+\frac{1}{k} \frac{\psi_{4,1} a}{\pi}+o\left(k^{-1}\right) \\
e^{-2 i \tau_{k} a}=e^{-2 i \tau_{k, 0} a} \exp \left(-2 i a\left(\frac{\tau_{k, 1}}{k}+o\left(k^{-1}\right)\right)\right)=-i-\frac{2 a \tau_{k, 1}}{k}+o\left(k^{-1}\right)
\end{gathered}
$$

Since $D_{1}\left(\mu_{k}\right)=0$ can be written as

$$
0=\mu_{k}^{-5} \psi_{1}\left(\mu_{k}\right)+\mu_{k}^{-5} \psi_{4}\left(\mu_{k}\right) e^{-2 i \tau_{k} a},
$$

comparison of coefficents of $k^{-1}$ leads to

$$
\tau_{k, 1}=\frac{\psi_{1,1}-\psi_{4,1} i}{\pi 4 \alpha(1-i)}=\frac{1}{8 \pi \alpha}\left(\psi_{1,1}+\psi_{4,1}+i\left(\psi_{1,1}-\psi_{4,1}\right)\right)
$$

A lengthy but straightforward calculation leads to

$$
\psi_{1,1}=4 \alpha\left(\varphi_{1}(a)-\varphi_{1}(0)\right)+4, \quad \psi_{4,1}=4 \alpha\left(\varphi_{1}(a)-\varphi_{1}(0)\right)-4 .
$$


Therefore

$$
\tau_{k, 1}=\frac{1}{8 \pi \alpha}\left(8 \alpha\left(\varphi_{1}(a)-\varphi_{1}(0)\right)+8 i\right)=\frac{1}{\pi}\left(\varphi_{1}(a)-\varphi_{1}(0)\right)+\frac{i}{\pi \alpha} .
$$

Finally we need to find the function $\varphi_{1}$. According to $[7,(8.2 .45)]$,

$$
\varphi_{1}=\varepsilon^{\top} \widehat{Q}^{[1]} e_{1}
$$

where $\varepsilon^{\boldsymbol{\top}}=(1,1,1,1), e_{1}^{\boldsymbol{\top}}=(1,0,0,0)$, and the $4 \times 4$ matrix function $\widehat{Q}^{[1]}$ is a solution of (see $[7,(8.2 .33)$ and $(8.2 .34)]$ - note that $\widehat{Q}^{[0]}=I_{4}$ by $[7,(8.2 .18)]$ )

$$
\begin{aligned}
\Omega_{4} Q^{[1]}-Q^{[1]} \Omega_{4} & =0 \\
Q_{\nu, \nu}^{[1]}{ }^{\prime}+\frac{1}{4}(-g) e_{\nu}^{\top} \Omega_{4} \varepsilon \varepsilon^{\top} \Omega_{4}^{-2} e_{\nu} & =0, \quad \nu=1, \ldots, 4 .
\end{aligned}
$$

Here $\Omega_{4}=\operatorname{diag}(1, i,-1,-i)$. Clearly, (5.8) implies that $\widehat{Q}^{[1]}$ is a diagonal matrix, whence $\varphi_{1}=\widehat{Q}_{11}^{[1]}$ and thus $\varphi_{1}^{\prime}-\frac{1}{4} g=0$, i. e., we can take

$$
\varphi_{1}(x)=\frac{1}{4} \int_{0}^{x} g(t) d t
$$

Theorem 5.2. For $g \in C^{1}[0, a]$, there is a positive integer $k_{0}$ such that the eigenvalues $\lambda_{k}, k \in \mathbb{Z}$, of the problem (1.1)-(1.5), counted with multiplicity, can be enumerated in such a way that the eigenvalues $\lambda_{k}$ are pure imaginary for $|k|<k_{0}, \lambda_{-k}=-\overline{\lambda_{k}}$ for $k \geq k_{0}$, where $\lambda_{k}=\mu_{k}^{2}$ with

$$
\mu_{k}=k \frac{\pi}{a}+\frac{\pi}{4 a}+\frac{1}{k} \frac{1}{\pi}\left(\frac{1}{4} \int_{0}^{a} g(t) d t+\frac{i}{\alpha}\right)+O\left(k^{-2}\right)
$$

as $k \rightarrow \infty$. In particular, there is an odd number of pure imaginary eigenvalues.

\section{The real and pure imaginary eigenvalues}

First pure imaginary eigenvalues will be investigated.

Lemma 6.1. It holds:

1. There is a number $\tau^{-}>0$ such that for all $\tau \geq \tau^{-}$and $\alpha>0,-i \tau$ is not an eigenvalue of the pencil $L(\lambda, \alpha)$.

2. There is an eigenvalue i $\tau$ of the pencil $L(\lambda, \alpha)$ on the imaginary axis with the asymptotics $\tau=\frac{2}{\alpha^{2}}+O\left(\frac{1}{\alpha}\right)$ as $\alpha \searrow 0$, whereas all other eigenvalues on the imaginary axis remain uniformly bounded as $\alpha \searrow 0$. 
Proof. 1. In the notations of $\S 5$, let $\mu=(1-i) \gamma, \gamma>0$. Then $\lambda=\mu^{2}=-2 i \gamma^{2}$. Since $\arg \mu=-\frac{\pi}{4} \in\left[-\frac{3 \pi}{8}, \frac{\pi}{8}\right]$, we have to show that there is $\gamma_{0}$ such that $D_{1}$ given by $(5.3)$ has no zeros $D_{1}((1-i) \gamma)=0$ for $\gamma>\gamma_{0}$. Observe that

$$
e^{-2 i \mu a}=e^{-2 i(1-i) \gamma a}=e^{-2(i+1) \gamma a}=O\left(\gamma^{-2}\right)
$$

so that with (5.5), (5.7), and (5.10),

$$
\begin{aligned}
{[(1-i) \gamma]^{-5} D_{1}((1-i) \gamma) } & =2 \alpha(1+i)+\frac{4 \alpha\left(\varphi_{1}(a)-\varphi_{1}(0)\right)+4}{(1-i) \gamma}+O\left((1+\alpha) \gamma^{-2}\right) \\
& =\frac{2}{\gamma}(1+i)\left[\alpha \gamma+\alpha\left(\varphi_{1}(a)-\varphi_{1}(0)\right)+1+O\left((1+\alpha) \gamma^{-1}\right)\right]
\end{aligned}
$$

The statement follows by choosing $\gamma_{0}>\left|\varphi_{1}(a)-\varphi_{1}(0)\right|+1$ such that the absolute value of the remainder term $O\left((1+\alpha) \gamma^{-1}\right)$ is bounded by $\frac{1}{2}(1+\alpha)$ for $\gamma>\gamma_{0}$.

2. Observe that replacing $\lambda$ by $-\lambda$ and $\alpha$ by $-\alpha$ does not change the eigenvalue problem and that the symmetry in Lemma 3.1 as well as the results in $\S 5$ also hold for $\alpha<0$. With $\mu=(1-i) \gamma, \gamma>0$ and $\lambda=-\mu^{2}=2 i \gamma^{2}$, the eigenvalue equation therefore reads

$$
-\alpha \gamma-\alpha\left(\varphi_{1}(a)-\varphi_{1}(0)\right)+1+O\left((1+\alpha) \gamma^{-1}\right)=0
$$

Although $D_{1}$ is not an entire function, we can argue as in $\S 5$ that there is an entire function which satisfies the same representation as above. In particular, by Rouché's theorem, $D_{1}$ has exactly one simple zero

$$
\gamma=\frac{1}{\alpha}-\varphi_{1}(a)+\varphi_{1}(0)+O(1)
$$

which also satisfies

$$
\gamma=\frac{1}{\alpha}-\varphi_{1}(a)+\varphi_{1}(0)+O(\alpha)
$$

and there is no other $\gamma(\alpha)$ with $\gamma(\alpha)>0$ and $\gamma(\alpha) \rightarrow \infty$ as $\alpha \searrow 0$. Thus

$$
\lambda_{k}=2 i\left(\frac{1}{\alpha}-\varphi_{1}(a)+\varphi_{1}(0)+O(\alpha)\right)^{2}=2 i \frac{1}{\alpha^{2}}-4 i \frac{1}{\alpha}\left(\varphi_{1}(a)-\varphi(0)\right)+O(1)
$$

is the only eigenvalue of $L(\lambda, \alpha)$ in a disc about $2 i\left(\frac{1}{\alpha}-\varphi_{1}(a)+\varphi_{1}(0)\right)^{2}$ whose radius can be chosen to be a positive multiple of $\frac{1}{\alpha}$. For sufficiently large $\frac{1}{\alpha}$, both $\lambda_{k}$ and $-\overline{\lambda_{k}}$ belong to this disc, and each must be an eigenvalue of $L(\lambda, \alpha)$ by the symmetry of the eigenvalues. This implies $\lambda_{k}=-\overline{\lambda_{k}}$, and therefore $\lambda_{k}=i \tau$ with $\tau=\frac{2}{\alpha^{2}}+O\left(\frac{1}{\alpha}\right) \in \mathbb{R}$. 
Note that the behavior of all eigenvalues in the upper half-plane is "discontinuous" as $\alpha$ approaches zero since Theorem 5.2 gives that $\operatorname{Im} \lambda_{k}=\frac{1}{\alpha a}+O\left(\frac{1}{k}\right)$.

Using Lemmas 3.1, 3.4, and 6.1 and in particular the fact that no eigenvalues can leave or join 0 from below on the imaginary axis as $\alpha$ varies in $(0, \infty)$, we arrive at

Theorem 6.2. It holds:

1. If the pencil $L(\lambda, 0)$ has eigenvalues on the negative imaginary axis and if $i \tau_{0}, \tau_{0}<0$ is the pure imaginary eigenvalue of $L(\lambda, 0)$ with the smallest imaginary part, then all pure imaginary eigenvalues i $\tau$ of the operator pencil $L(\lambda, \alpha)$ for $\alpha>0$ satisfy $\tau \geq \tau_{0}$.

2. The number $\kappa$ of eigenvalues of the pencil $L(\lambda, \alpha), \alpha \geq 0$, on the negative imaginary axis, (counted with multiplicity) is independent of $\alpha$.

3. If $\alpha \searrow 0$, one eigenvalue leaves the positive imaginary axis through $+i \infty$, whereas no eigenvalue leaves the negative imaginary axis.

Theorem 6.3. The eigenvalues of the operator pencil $L(\lambda, \alpha), \alpha>0$, possess the following properties:

1. All the eigenvalues lie in the closed upper half-plane and on the imaginary axis and are symmetric with respect to the imaginary axis.

2. The number of real eigenvalues is finite, and the number of pure imaginary eigenvalues is finite and odd, counted with multiplicity.

3. All (if any) of the nonzero real eigenvalues are simple.

4. If $g \geq 0$, then all eigenvalues lie in the closed upper half-plane.

Proof. For 1. see Lemma 3.1; 2. follows from the asymptotics in Theorem 5.2.

To prove Statement 3 we note that nonzero real eigenvalues are semisimple by Lemma 3.2. Thus it remains to prove that nonzero real eigenvalues are geometrically simple. Denote by $y_{j}(\lambda, x)$ the solution of (1.1) which satisfies the conditions $y_{j}^{(m)}(\lambda, 0)=\delta_{j, m+1}$ for $j=1, \ldots, 4, m=0, \ldots, 3$. The general solution of (1.1) which satisfies (1.2) and (1.3) is given by

$$
y=c_{1} y_{2}(\lambda, x)+c_{2} y_{4}(\lambda, x) .
$$

Hence, if $\lambda$ were not simple, then (6.2) would be the general solution of (1.1)(1.5), and thus (1.4) and (1.5) and the fact that $y_{j}(\lambda, \cdot)$ is real would lead to

$$
y_{j}(\lambda, a)=0, \quad y_{j}^{\prime}(\lambda, a)=0, \quad y_{j}^{\prime \prime}(\lambda, a)=0
$$

for $\lambda=1,2$. But the set of solutions of (1.1) satisfying (6.3) is one-dimensional, which contradicts the assumption that $\lambda$ is not simple.

For the proof of Statement 4 note that $g \geq 0$ implies $A \geq 0$. Any eigenvalue $-i \tau$ with $\tau>0$ with corresponding eigenvector $Y_{0}$ would satisfy (3.7), and hence

$$
-\tau^{2}\left(M Y_{0}, Y_{0}\right)-\tau \alpha\left(K Y_{0}, Y_{0}\right)-\left(A Y_{0}, Y_{0}\right)=0,
$$


which is impossible because of $\tau>0, \alpha \geq 0, K \geq 0, A \geq 0$, and $\left.M\right|_{D(A)}>0$.

Proposition 6.4. Let $\lambda_{k}\left(\alpha_{0}\right)=-i \tau$ and $\lambda_{n}\left(\alpha_{0}\right)=i \tau\left(\alpha_{0}>0, \tau>0\right)$ be eigenvalues of $L\left(\lambda, \alpha_{0}\right)$. Then they are eigenvalues of $L(\lambda, \alpha)$ for all $\alpha \geq 0$ and at most one of them can be multiple. In particular, if $\lambda\left(\alpha_{0}\right)$ is a double eigenvalue of $L\left(\lambda, \alpha_{0}\right)$ on the negative imaginary axis, then also $-\lambda\left(\alpha_{0}\right)$ is an eigenvalue of $L\left(\lambda, \alpha_{0}\right)$.

Proof. The set $S$ of solutions of (1.1) with $\lambda^{2}=-\tau^{2}$ satisfying the boundary conditions (1.2)-(1.4) is a vector space of dimension 1 or 2 . If the dimension of $S$ is 2 , then for each $\alpha \in \mathbb{R}$ it has at least one nonzero element $y$ such that also

$$
y^{\prime \prime}(a)+\alpha \tau y^{\prime}(a)=0 .
$$

This means that $-i \tau$ and $i \tau$ are eigenvalues of $L(\lambda, \alpha)$ for all $\alpha \geq 0$. In particular, this case prevails if $\lambda$ has geometric multiplicity 2, proving the last statement of Proposition 6.4. If the dimension of $S$ is 1 , then $S$ must be the eigenspace for both $\lambda=-i \tau$ and $\lambda=i \tau$, and choosing a nonzero $y \in S$, this would be the first component of an eigenvector for both eigenvalues of $L(\lambda, \alpha)$. Then (1.5), applied to both $\lambda=i \tau$ and $\lambda=-i \tau$, gives

$$
y^{\prime \prime}(a)-\alpha_{0} \tau y^{\prime}(a)=0=y^{\prime \prime}(a)+\alpha_{0} \tau y^{\prime}(a) .
$$

Clearly, $y^{\prime \prime}(a)=0=y^{\prime}(a)$ follows, and hence

$$
y^{\prime \prime}(a) \pm \alpha \tau y^{\prime}(a)=0
$$

for $\alpha \geq 0$. This proves that $i \tau$ and $-i \tau$ are eigenvalues of $L(\lambda, \alpha)$ for all $\alpha \geq 0$.

If both eigenvalues $-i \tau$ and $i \tau$ were geometrically double, then $\operatorname{dim} S=2$ and $S$ would be the eigenspace for both $-i \tau$ and $i \tau$. In particular,

$$
y(a)=y^{\prime \prime}(a)+\alpha \tau y^{\prime}(a)=y^{\prime \prime}(a)-\alpha \tau y^{\prime}(a)=0
$$

for all $y \in S$. But this leads to $y(a)=y^{\prime}(a)=y^{\prime \prime}(a)$ for all $y \in S$, which is impossible since the solutions of the fourth order differential (1.1) satisfying these initial conditions form a onedimensional space. Note that if one of the eigenvalues $-i \tau, i \tau$ is geometrically double, then $y \in S$ with $y^{\prime}(a)=0$ is the first component of an eigenfunction for all $\alpha$, and therefore the first components $y$ of the eigenfunctions of the geometric simple eigenvalue must satisfy $y^{\prime}(a)=0$.

Finally we show that when $-i \tau$ is (geometrically) double then $i \tau$ is algebraically simple. Suppose that the eigenvalue $i \tau$ has an associated vector $Y_{1}$, and denote the eigenvector by $Y_{0}$. Then $2 i \tau\left(M Y_{0}, Y_{0}\right)-i \alpha_{0}\left(K Y_{0}, Y_{0}\right)=0$, i. e.,

$$
2 \tau \int_{0}^{a}|y(x)|^{2} d x-\alpha_{0}\left|y^{\prime}(a)\right|^{2}=0
$$

where $y$ is the first component of $Y_{0}$. We have seen in the previous paragraph that $y^{\prime}(a)=0$. Thus (6.4) leads to the contradiction $y=0$. 
Recall from Theorem 5.2 that the pure imaginary eigenvalues of the operator pencil $L(\lambda, \alpha)$ have been denoted by $\lambda_{k}$ with $|k|<k_{0}$. The following theorem is concerned with these eigenvalues.

Theorem 6.5. The pure imaginary eigenvalues of the operator pencil $L(\lambda, \alpha)$, $\alpha>0$, can be split into two finite sequences $\lambda_{k}^{(1)}\left(k=-\kappa_{1}, \ldots, 2\left(k_{0}-p-1\right)-\kappa_{1}\right)$ and $\lambda_{k}^{(2)}(k= \pm 1, \ldots, \pm p)$ for some nonnegative integers $\kappa_{1}$ and $p$, having the following properties:

1. The eigenvalues $\lambda=i \tau \in i \mathbb{R}$ with $\tau<0$ are semisimple with algebraic multiplicities 1 or 2 .

2. The eigenvalues $\lambda_{k}^{(1)}$ for $k=-1,-2, \ldots,-\kappa_{1}$ and $\lambda_{k}^{(2)}$ for $k=-1,-2, \ldots$, $-p$ (if any) are exactly the eigenvalues in $(-i \infty, 0)$, counted with multiplicity. The eigenvalues $\lambda_{k}^{(2)}$ for $k=-1,-2, \ldots,-p$ are independent of $\alpha$ and $\lambda_{k}^{(2)}=-\lambda_{-k}^{(2)}$ for $k=-1,-2, \ldots,-p$.

3. We assume the eigenvalues $\lambda_{k}^{(1)}$ and $\lambda_{k}^{(2)}$ are arranged in nondecreasing order along the imaginary axis: $\operatorname{Im} \lambda_{k}^{(j)} \geq \operatorname{Im} \lambda_{n}^{(j)}$ for $k>n$ and $j=1,2$. Then $0>\operatorname{Im} \lambda_{-1}^{(1)}>\operatorname{Im} \lambda_{-2}^{(1)}>\cdots>\operatorname{Im} \lambda_{-\kappa_{1}}^{(1)}$ and $\operatorname{Im} \lambda_{-p}^{(2)}<\operatorname{Im} \lambda_{-p+1}^{(2)}<$ $\cdots<\operatorname{Im} \lambda_{-1}^{(2)}<0<\operatorname{Im} \lambda_{1}^{(2)}<\cdots<\operatorname{Im} \lambda_{p}^{(2)}$.

4. If $\kappa_{1}>0$, then none of the numbers $i\left|\lambda_{-j}^{(1)}\right|\left(j=1, \ldots, \kappa_{1}\right)$ equals a term of the sequence $\lambda_{k}^{(1)}\left(k=-\kappa_{1}, \ldots, 2\left(k_{0}-p-1\right)-\kappa_{1}\right)$.

5. If $\kappa_{1}>1$, then the number of terms of the sequence $\lambda_{k}^{(1)}$ in the intervals $\left(i\left|\lambda_{-j}^{(1)}\right|, i\left|\lambda_{-(j+1)}^{(1)}\right|\right)\left(j=1, \ldots, \kappa_{1}-1\right)$ is odd.

6. If $\kappa_{1}>0$, then the number of terms of the sequence $\lambda_{k}^{(1)}$ in the interval $\left[0, i\left|\lambda_{-1}^{(1)}\right|\right)$ is even or zero.

7. If $\kappa_{1}>0$, then the number of terms of the sequence $\lambda_{k}^{(1)}$ in the interval $\left(i\left|\lambda_{-\kappa_{1}}^{(1)}\right|, i \infty\right)$ is even or zero.

8. If $\kappa_{1}=0$, then the sequence $\lambda_{k}^{(1)}$ has an odd number of terms, which lie on the nonnegative imaginary axis.

Proof. 1. By Lemma 3.3 these eigenvalues are semisimple, and (1.2), (1.3) imply that the geometric multiplicity of any eigenvalue cannot exceed 2 .

2. We extract the symmetric eigenvalues $-i \tau, i \tau$ for $\tau>0$ from the sequence of $\lambda_{k},|k|<k_{0}$ and denote them by $\lambda_{k}^{(2)}$, where $p$ is the number of $\tau>0$ for which both $i \tau$ and $-i \tau$ are eigenvalues. The remaining part of 2 . and the statement 3. follow by suitable indexing and from Proposition 6.4.

4. If $i\left|\lambda_{-j}^{(1)}\right|$ is an eigenvalue of $L(\lambda, \alpha)$, then one copy of $\lambda_{-j}^{(1)}$ and $i\left|\lambda_{-j}^{(1)}\right|$ is moved to the sequence of $\lambda_{k}^{(2)}$, and $\lambda_{-j}^{(1)}$ as well as $i\left|\lambda_{-j}^{(1)}\right|$ would be multiple eigenvalues, which is impossible by Proposition 6.4. 
5. By Lemma 3.4, eigenvalues on the negative imaginary axis are nondecreasing in the parameter $\alpha$. In particular, an eigenvalue on the negative imaginary axis $\lambda_{k}^{(1)}=\lambda_{k}^{(1)}(\alpha)$ belongs to a continuous branch of eigenvalues, for which $\lambda_{k}^{(1)}(0)$ is therefore also an eigenvalue on the negative imaginary axis. But for $\alpha=0$ the eigenvalues are symmetric. Note that if an eigenvalue $\lambda$ (on the negative imaginary axis) has an eigenfunction $y$ with $y^{\prime}(a)=0$, then it will be an eigenvalue for all $\alpha$. Hence $\lambda=\lambda_{j}^{(2)}$ and $\lambda$ has geometric multiplicity 2 . If all eigenfunctions $y$ would satisfy $y^{\prime}(a)=0$, then $\lambda$ and $-\lambda$ would have geometric multiplicity 2, which is impossible by Proposition 6.4. Therefore, if $\lambda=\lambda_{k}^{(1)}$, there is an eigenfunction $y$ with $y^{\prime}(a) \neq 0$, and by (3.11), this implies that $\dot{\lambda}_{k}^{(1)}>0$ (in particular for $\alpha=0$ ). ¿From statement 1 of Lemma 3.4 it therefore follows that the eigenvalue $i\left|\lambda_{k}^{(1)}(0)\right|$ becomes an eigenvalue $\lambda_{n}^{(1)}(\alpha)$ on the positive imaginary axis for small $\alpha>0$ with $\left|\lambda_{n}^{(1)}(\alpha)\right|>\left|\lambda_{k}^{(1)}(\alpha)\right|$. This proves 5 . for small $\alpha$ (with the odd number being 1). As $\alpha$ increases, eigenvalues $\lambda_{n}^{(1)}$ on the positive imaginary axis can never cross the (moving) numbers $i\left|\lambda_{k}^{(j)}\right|$ with $\lambda_{k}^{(j)}$ on the negative imaginary axis, see Proposition 6.4. Also, eigenvalues can leave and join the nonnegative imaginary axis only in pairs.

7. follows as for 5 . if we observe that, for $\alpha>0$, an odd number of eigenvalues moves onto the imaginary axis from $i \infty$ by Theorem 6.23 . Statements 6. and 8. follow from the other statements since the number of $\lambda_{k}^{(1)}$ is odd.

We do not know if double eigenvalues on the negative imaginary axis exist.

In the next section we will see that for constant $g, L(\lambda, \alpha)$ does not have nonzero real eigenvalues. However, for nonconstant $g$ we have:

Example 6.6. There are functions $y \in C^{\infty}[0,1]$ with $y>0$ on $(0,1), y(0)=$ $y^{\prime \prime}(0)=y(1)=y^{\prime}(1)=y^{\prime \prime}(1)=y^{(4)}(1)=0, y^{\prime}(0) \neq 0, y^{(3)}(1) \neq 0, y^{\prime}$ has only one zero, say $x_{0}=\frac{1}{2}$, in $(0,1)$, which is simple, and $y^{(3)}(1)-y^{(3)}\left(x_{0}\right)>0$. Let

$$
\begin{aligned}
\lambda & :=\left(y^{(3)}(1)-y^{(3)}\left(x_{0}\right)\right)^{\frac{1}{2}}\left(\int_{x_{0}}^{1} y(x) d x\right)^{-\frac{1}{2}} \\
g(x) & :=\left(y^{(3)}(x)-y^{(3)}(1)+\lambda^{2} \int_{x}^{1} y(t) d t\right) \frac{1}{y^{\prime}(x)} .
\end{aligned}
$$

Then $g \in C^{\infty}[0,1]$ and $\lambda$ is a positive real eigenvalue of (1.1)-(1.5).

\section{Real and pure imaginary eigenvalues for constant $g$}

Let $\alpha>0$. We divide our investigation into various subcases; a summary will be given in the next section. The characteristic equation of the differential equation (1.1) is

$$
z^{4}-g z^{2}-\lambda^{2}=0
$$


which gives the characteristic exponents

$$
z= \pm \sqrt{\frac{g}{2} \pm \sqrt{\frac{g^{2}}{4}+\lambda^{2}}}
$$

where for definiteness we use the argument in $[0, \pi)$ for the square root.

7.1. Real eigenvalues for $g>0$. Here we consider $g>0$ and $\lambda \in \mathbb{R}$.

First let $\lambda=0$. Then the characteristic equation has simple zeros at $\pm \sqrt{g}$ and a double zeros at 0 , and the solution of (1.1) subject to $y(0)=y^{\prime \prime}(0)=0$ is

$$
y(x)=c_{1} \sinh (\sqrt{g} x)+c_{2} x .
$$

Substituting the boundary conditions (1.4) and (1.5) gives $c_{1}=c_{2}=0$, and thus $\lambda=0$ is not an eigenvalue.

If now $\lambda \neq 0$, then the four characteristic exponents are $\pm \nu_{1}, \pm i \nu_{2}$, where $0<\nu_{2}<\nu_{1}$, and the general solution of (1.1) subject to $y(0)=y^{\prime \prime}(0)=0$ is

$$
y(x)=c_{1} \sinh \left(\nu_{1} x\right)+c_{2} \sin \left(\nu_{2} x\right) .
$$

Again substituting (1.4) and (1.5), the determinant of the matrix of the resulting $2 \times 2$ system for $c_{1}, c_{2}$ has real and imaginary parts

$$
\begin{gathered}
-\left(\nu_{1}^{2}+\nu_{2}^{2}\right) \sinh \left(\nu_{1} a\right) \sin \left(\nu_{2} a\right), \\
\alpha \lambda\left(\nu_{2} \sinh \left(\nu_{1} a\right) \cos \left(\nu_{2} a\right)-\nu_{1} \cosh \left(\nu_{1} a\right) \sin \left(\nu_{2} a\right)\right),
\end{gathered}
$$

at least one of which is nonzero. Thus $\lambda$ is no eigenvalue.

7.2. Real eigenvalues for $g<0$. Here we consider $g<0$ and $\lambda \in \mathbb{R}$.

For $\lambda=0$ the characteristic equation has simple zeros at $\pm i \sqrt{|g|}$ and a double zeros at 0 , and the solution of (1.1) subject to $y(0)=y^{\prime \prime}(0)=0$ is

$$
y(x)=c_{1} \sin (\sqrt{|g|} x)+c_{2} x .
$$

Substituting (1.4) and (1.5) gives $c_{2}=0$, and a nontrivial solution exists if and only if $\sin (\sqrt{|g|} a)=0$, i. e., if and only if $\sqrt{|g|} a=k \pi$ for a positive integer $k$. This eigenvalue is geometrically simple. To show that it is algebraically simple assume, by contradiction, that there is an associated function $y_{1}$. Then, see (3.1) and (3.2), $y_{1}$ satisfies the same differential equation and the same boundary conditions as $y$, with the exception that $y_{1}^{\prime \prime}(a)=0$ is replaced by $y_{1}^{\prime \prime}(a)+$ $i \alpha y^{\prime}(a)=0$. But then $y_{1}(x)$ must be a multiple of $\sin (\sqrt{|g|} x)$, and $y^{\prime}(a) \neq 0$, $y_{1}^{\prime \prime}(a)=0$ shows that this last boundary condition is not satisfied.

If now $\lambda \neq 0$, then the four characteristic exponents are $\pm \nu_{1}, \pm i \nu_{2}$, where $0<\nu_{1}<\nu_{2}$, and the general solution of (1.1) subject to $y(0)=y^{\prime \prime}(0)=0$ is

$$
y(x)=c_{1} \sinh \left(\nu_{1} x\right)+c_{2} \sin \left(\nu_{2} x\right) .
$$

Similar to the case $g>0$ it follows that $\lambda$ is no eigenvalue. 
7.3. Pure imaginary eigenvalues for $g<0$. Here we consider $\lambda \in i \mathbb{R}, \lambda \neq 0$. Putting $\lambda=i \tau, \tau \in \mathbb{R} \backslash\{0\}$, the characteristic exponents become

$$
z= \pm \sqrt{\frac{g}{2} \pm \sqrt{\frac{g^{2}}{4}-\tau^{2}}}
$$

We have to distinguish three cases: $|\tau|<\frac{|g|}{2},|\tau|=\frac{|g|}{2}$, and $|\tau|>\frac{|g|}{2}$.

Case I: $\tau= \pm \frac{|g|}{2}$. In this case, $\mu= \pm i \sqrt{\frac{|g|}{2}}=: \pm i \nu$, both double characteristic exponents, $\nu>0$. The general solution of $(1.1)$ subject to $y(0)=y^{\prime \prime}(0)=0$ is

$$
y(x)=c_{1} \sin (\nu x)+c_{2} x \cos (\nu x) .
$$

Substituting (1.4) and (1.5), the determinant of the matrix of the resulting $2 \times 2$ system for $c_{1}, c_{2}$ is

$$
\begin{aligned}
& \sin (\nu a)\left[-2 \nu \sin (\nu a)-\nu^{2} a \cos (\nu a)-\alpha \tau \cos (\nu a)+\alpha \tau \nu a \sin (\nu a)\right] \\
& -a \cos (\nu a)\left[-\nu^{2} \sin (\nu a)-\alpha \tau \nu \cos (\nu a)\right] \\
= & -2 \nu \sin ^{2}(\nu a)+\alpha \tau(\nu a-\sin (\nu a) \cos (\nu a)) .
\end{aligned}
$$

Note that this term is always negative if $\tau<0$, so that there are no eigenvalues $\lambda=-i \frac{|g|}{2}$. If $\tau>0$, then $-2 \nu \sin ^{2}(\nu a)$ dominates for $g$ close to 0 , whereas $\alpha \tau \nu a$ dominates for large $|g|$ (note that $\tau$ and $\nu$ depend on $g$ ), so that there is an odd number of $g \in(-\infty, 0)$ for which $\lambda=i \frac{|g|}{2}$ is an eigenvalue.

Case II: $0<|\tau|<\frac{g}{2}$. Here $z= \pm i \nu_{1}, \pm i \nu_{2}$, where $0<\nu_{2}<\nu_{1}$. The general solution of $(1.1)$ subject to $y(0)=y^{\prime \prime}(0)=0$ is

$$
y(x)=c_{1} \sin \left(\nu_{1} x\right)+c_{2} \sin \left(\nu_{2} x\right) .
$$

Substituting (1.4) and (1.5), the determinant of the matrix of the resulting $2 \times 2$ system for $c_{1}, c_{2}$ is

$$
\begin{aligned}
& \sin \left(\nu_{1} a\right)\left[-\nu_{2}^{2} \sin \left(\nu_{2} a\right)-\alpha \tau \nu_{2} \cos \left(\nu_{2} a\right)\right]-\sin \left(\nu_{2} a\right)\left[-\nu_{1}^{2} \sin \left(\nu_{1} a\right)-\alpha \tau \nu_{1} \cos \left(\nu_{1} a\right)\right] \\
& =\left(\nu_{1}^{2}-\nu_{2}^{2}\right) \sin \left(\nu_{1} a\right) \sin \left(\nu_{2} a\right)-\alpha \frac{\tau}{a}\left(\nu_{2} a \sin \left(\nu_{1} a\right) \cos \left(\nu_{2} a\right)-\nu_{1} a \cos \left(\nu_{1} a\right) \sin \left(\nu_{2} a\right)\right) .
\end{aligned}
$$

Since both terms are oscillatory, we have, both for $\tau>0$ and $\tau<0$, a finite number of eigenvalues $i \tau$ with $0<|\tau|<\frac{|g|}{2}$, where the number of eigenvalues with $\tau>0$ and $\tau<0$, respectively, is positive for sufficiently large $|g|$.

Both $i \tau$ and $-i \tau$ are eigenvalues for some $\tau \in\left(0, \frac{|g|}{2}\right)$ if and only if $\sin \left(\nu_{1} a\right)=$ $\sin \left(\nu_{2} a\right)=0$, i. e., if $\nu_{j}=\frac{k_{j} \pi}{a}$, where $k_{2}<k_{1}$ are positive integers. Thus

$$
\frac{k_{1}^{2} \pi^{2}}{a^{2}}=\frac{|g|}{2}+\sqrt{\frac{g^{2}}{4}-\tau^{2}}, \quad \frac{k_{2}^{2} \pi^{2}}{a^{2}}=\frac{|g|}{2}-\sqrt{\frac{g^{2}}{4}-\tau^{2}},
$$


which is equivalent to

$$
g=-\left(k_{1}^{2}+k_{2}^{2}\right) \frac{\pi^{2}}{a^{2}}, \quad \tau=k_{1} k_{2} \frac{\pi^{2}}{a^{2}} .
$$

Hence both $i \tau$ and $-i \tau$ with $0<\tau<\frac{|g|}{2}$ are eigenvalues if and only if there are distinct positive integers $k_{1}$ and $k_{2}$ such that (7.2) holds.

We will show that these symmetric eigenvalues are simple. To this end let

$$
\begin{aligned}
f(\tau)= & \left(\nu_{1}^{2}-\nu_{2}^{2}\right) \sin \left(\nu_{1} a\right) \sin \left(\nu_{2} a\right) \\
& -\alpha \frac{\tau}{a}\left(\nu_{2} a \sin \left(\nu_{1} a\right) \cos \left(\nu_{2} a\right)-\nu_{1} a \cos \left(\nu_{1} a\right) \sin \left(\nu_{2} a\right)\right) .
\end{aligned}
$$

At $g=-\left(k_{1}^{2}+k_{2}^{2}\right) \frac{\pi^{2}}{a^{2}}, \tau=k_{1} k_{2} \frac{\pi^{2}}{a^{2}}$, we have

$$
f^{\prime}(\tau)=-\alpha \tau a\left(\nu_{2} \frac{d \nu_{1}}{d \tau}-\nu_{1} \frac{d \nu_{2}}{d \tau}\right) \cos \left(\nu_{1} a\right) \cos \left(\nu_{2} a\right)
$$

Thus if any of those eigenvalues were not simple, then $\frac{d\left(\nu_{1} / \nu_{2}\right)}{d \tau}=0$, which is impossible since

$$
\frac{\nu_{1}^{2}}{\nu_{2}^{2}}=\frac{\frac{|g|}{2}+\sqrt{\frac{g^{2}}{4}-\tau^{2}}}{\frac{|g|}{2}-\sqrt{\frac{g^{2}}{4}-\tau^{2}}}=\frac{\frac{g^{2}}{2}-\tau^{2}+|g| \sqrt{\frac{g^{2}}{4}-\tau^{2}}}{\tau^{2}}=\frac{\frac{g^{2}}{2}+|g| \sqrt{\frac{g^{2}}{4}-\tau^{2}}}{\tau^{2}}-1
$$

is strictly decreasing as a function of $\tau^{2}$.

Case III: $|\tau|>\frac{g}{2}$. In this case, we can write $z= \pm \nu \pm i \kappa$ with $0<\nu<\kappa$. The general solution of (1.1) subject to $y(0)=y^{\prime \prime}(0)=0$ is

$$
y(x)=c_{1} \cosh (\nu x) \sin (\kappa x)+c_{2} \sinh (\nu x) \cos (\kappa x) .
$$

Using the notations $s_{\nu}=\sinh (\nu a), c_{\nu}=\cosh (\nu a), s_{\kappa}=\sin (\kappa a), c_{\kappa}=\cos (\kappa a)$, the determinant $d$ of the coefficient matrix for $c_{1}, c_{2}$ in the system $y(a)=0$, $y^{\prime \prime}(a)-\alpha \tau y^{\prime}(a)=0$ can be written as

$$
\begin{aligned}
d & =d_{1}-\alpha \tau d_{2} \\
d_{1} & =c_{\nu} s_{\kappa}\left[\left(\nu^{2}-\kappa^{2}\right) s_{\nu} c_{\kappa}-2 \nu \kappa c_{\nu} s_{\kappa}\right]-s_{\nu} c_{\kappa}\left[\left(\nu^{2}-\kappa^{2}\right) c_{\nu} s_{\kappa}+2 \nu \kappa s_{\nu} c_{\kappa}\right] \\
& =-2 \nu \kappa\left(c_{\nu}^{2} s_{\kappa}^{2}+s_{\nu}^{2} c_{\kappa}^{2}\right)<0 \\
d_{2} & =c_{\nu} s_{\kappa}\left[\nu c_{\nu} c_{\kappa}-\kappa s_{\nu} s_{\kappa}\right]-s_{\nu} c_{\kappa}\left[\nu s_{\nu} s_{\kappa}+\kappa c_{\nu} c_{\kappa}\right] \\
& =\nu s_{\kappa} c_{\kappa}\left(c_{\nu}^{2}-s_{\nu}^{2}\right)-\kappa c_{\nu} s_{\nu}\left(s_{\kappa}^{2}+c_{\kappa}^{2}\right) \\
& =\nu s_{\kappa} c_{\kappa}-\kappa c_{\nu} s_{\nu} \\
& =\frac{\nu}{2} \sin (2 \kappa a)-\frac{\kappa}{2} \sinh (2 \nu a)<0 .
\end{aligned}
$$

Hence $d<0$ if $\tau<0$, and thus there are no eigenvalues at if if $\tau<0$. By Lemma 4.12 . there may be finitely many eigenvalues of the form $i \tau$ with $\tau>\frac{g}{2}$. 


\section{Properties of the eigenvalues for constant $g$}

Theorem 8.1. Let $g$ be constant, $g \neq 0, g \neq-\left(k_{1}^{2}+k_{2}^{2}\right) \frac{\pi^{2}}{a^{2}}$ for any $k_{1} \in \mathbb{N}$, $k_{2} \in \mathbb{N}, k_{1} \neq k_{2}$. In addition to the properties stated in Theorem 6.3 , the eigenvalues of $L(\lambda, \alpha)(\alpha>0)$ possess the following properties:

1. All the eigenvalues lie in the open upper half-plane and on the imaginary axis and are symmetric with respect to the imaginary axis.

2. All the eigenvalues in the closed lower half plane are simple, and their total number is zero if $g>0$ and $\kappa_{0}=\left\lfloor\sqrt{|g|} \frac{a}{\pi}\right\rfloor$ if $g<0$, where \lfloor\rfloor denotes the integer part. Denote these eigenvalues $\lambda_{j}^{0}\left(j=1,2, \ldots, \kappa_{0}\right)$ enumerated monotonically: $\left|\lambda_{1}^{0}\right|<\left|\lambda_{2}^{0}\right|<\cdots<\left|\lambda_{\kappa_{0}}^{0}\right|$.

3. All the points $i\left|\lambda_{j}^{0}\right|$ with $\lambda_{j}^{0} \neq 0$ are not eigenvalues.

4. If $\kappa_{0}>1$, each interval $\left(i\left|\lambda_{j}^{0}\right|, i\left|\lambda_{j+1}^{0}\right|\right)\left(j=1,2, \ldots, \kappa_{0}-1\right)$ contains an odd number (with account of multiplicities) of eigenvalues, and if $\kappa_{0}>0$, the intervals $\left(0, i\left|\lambda_{1}^{0}\right|\right)$ and $\left(i\left|\lambda_{-\kappa_{0}}^{0}\right|,+i \infty\right)$ each contain an even number (possibly zero) of eigenvalues.

Proof. 1. To prove 1. it is enough to combine the result of Theorem 6.31 . with the result of $\S 7.1$ and $\S 7.2$ on absence of nonzero real eigenvalues.

2. By 1., all eigenvalues in the closed lower half-plane lie on the imaginary axis. There are none if $g>0$, see $\S 7.1$. Now let $g<0$. By Lemmas 3.3, all eigenvalues $i \tau, \tau<0$ are semisimple, and from the exposition in $\S 7.3$ it follows that they are geometrically simple. Hence they are simple. In $\S 7.2$ we have seen that this is also true for the eigenvalue $\lambda=0$. By Theorem 6.2 2. and Lemma 3.43 ., the number $\kappa_{0}$ is independent of $\alpha \geq 0$, where for $\lambda=0$ and $\alpha=0$, only the geometric multiplicity is taken. For $\alpha=0$ the results from $\S 7.2$ and $\S 7.3$ can be extended to this case to show that $\kappa_{0}=\kappa_{1}+\kappa_{2}+\kappa_{3}$, where

$$
\begin{aligned}
\kappa_{1} & =\left\{\begin{array}{ll}
1 & \text { if } \sqrt{|g|} \in \mathbb{Z} \frac{\pi}{a} \\
0 & \text { otherwise, }
\end{array} \quad \kappa_{2}= \begin{cases}1 & \text { if } \sqrt{\frac{|g|}{2}} \in \mathbb{Z} \frac{\pi}{a} \\
0 & \text { otherwise, }\end{cases} \right. \\
\kappa_{3} & =\#\left\{\tau \in\left(0, \frac{|g|}{2}\right): \sqrt{\frac{|g|}{2} \pm \sqrt{\frac{g^{2}}{4}-\tau^{2}}} \in \mathbb{Z} \frac{\pi}{a}\right\} \\
& =\#\left[\left(\left(0, \sqrt{\frac{|g|}{2}}\right) \cup\left(\sqrt{\frac{|g|}{2}}, \sqrt{|g|}\right)\right) \cap \mathbb{Z} \frac{\pi}{a}\right] .
\end{aligned}
$$

3. It was proved in $\S 7.3$ that eigenvalues on the negative imaginary axis can only occur in Case II and that no pair of pure imaginary eigenvalues which are symmetric with respect to the origin can occur.

4. The statement 4 follows from 3. and Theorem 6.5 5., 6., 7. 
Now let us consider the case of the exceptional value $g=-\left(k_{1}^{2}+k_{2}^{2}\right) \frac{\pi^{2}}{a^{2}}$ for some $k_{1} \in \mathbb{N}$ and $k_{2} \in \mathbb{N}, k_{2}>k_{1}$. The eigenvalue $\lambda=-i k_{1} k_{2} \frac{\pi^{2}}{a^{2}}$ is double for $\alpha=0$, remains stationary for $\alpha>0$, but becomes simple. Since the eigenvalues on the negative imaginary axis move upwards or remain stationary by Lemma 3.43 , and since they are simple, the number of pure imaginary eigenvalues below $-i k_{1} k_{2} \frac{\pi^{2}}{a^{2}}$ is independent of $\alpha \geq 0$. Using the formulas in the proof of Theorem 8.12 , it is easy to see that this number is

$$
\kappa_{2}=\#\left[\left(\left(k_{1} \frac{\pi}{a}, \sqrt{\frac{|g|}{2}}\right) \cup\left(\sqrt{\frac{|g|}{2}}, k_{2} \frac{\pi}{a}\right)\right) \cap \mathbb{Z} \frac{\pi}{a}\right]=k_{2}-k_{1}-1 .
$$

Therefore we have in this case the following theorem.

Theorem 8.2. Let $g=-\left(k_{1}^{2}+k_{2}^{2}\right) \frac{\pi^{2}}{a^{2}}$ for some $k_{1} \in \mathbb{N}$ and $k_{2} \in \mathbb{N}, k_{2}>k_{1}$. In addition to the properties stated in Theorem 6.3, the eigenvalues of $L(\lambda, \alpha)$ $(\alpha>0)$ possess the following properties:

1. All the eigenvalues lie in the open upper half-plane and on the imaginary axis and are symmetric with respect to the imaginary axis.

2. All the eigenvalues in the closed lower half plane are simple. The total number of the eigenvalues in the closed lower half-plane is $\kappa_{0}=\left\lfloor\sqrt{k_{1}^{2}+k_{2}^{2}}\right\rfloor$. Denote these eigenvalues $\lambda_{j}^{0}\left(j=1,2, \ldots, \kappa_{0}\right)$ enumerated monotonically: $\left|\lambda_{1}^{0}\right|<\left|\lambda_{2}^{0}\right|<\ldots<\left|\lambda_{\kappa_{0}}^{0}\right|$.

3. Only the eigenvalue $\lambda_{\kappa_{0}-k_{2}+k_{1}}^{0}=-i k_{1} k_{2} \frac{\pi^{2}}{a^{2}}$ on the negative imaginary axis has a symmetrically located eigenvalue on the positive imaginary axis.

4. Each interval $\left(i\left|\lambda_{j}^{0}\right|, i\left|\lambda_{j+1}^{0}\right|\right]\left(j=1,2, \ldots, \kappa_{0}-1\right)$ contains an odd number (counted with multiplicities) of eigenvalues, and the intervals $\left(0, i\left|\lambda_{1}^{0}\right|\right)$ and $\left(i\left|\lambda_{\kappa_{0}}^{0}\right|,+i \infty\right)$ each contain an even number (possibly zero) of eigenvalues.

Acknowledgement. The second author was supported in part by grants of the CRDF, U.S.A., under grant number UM1-2567-OD-03 and of the NRF of South Africa under grant number 2053746.

\section{References}

[1] Chandrasekhar, S., On characteristic value problems in high order differential equations which arise in studies on hydrodynamic and hydromagnetic stability. Amer. Math. Monthly 61, (1954)(7), part II, $32-45$.

[2] Davies, H. T., An extension of the problem of the elastic bar. Amer. J. Math. $47(1925)(2), 101-120$.

[3] Eni, V. M., Stability of the root number of an analytic operator function and on perturbations of its characteristic numbers and eigenvectors (in Russian). Dokl. Akad. Nauk SSSR 173 (1967), 1251 - 1254. 
[4] Geist, B. and McLaughlin, J. R., Double eigenvalues for the uniform Timoshenko beam. Appl. Math. Lett. 10 (1997)(3), 129 - 134.

[5] Kato, T., Perturbation Theory for Linear Operators (2nd ed.). New York: Springer 1976.

[6] Kostyuchenko, A. G. and Orazov, M. B., The problem of oscillations of an elastic half cylinder and related selfadjoint quadratic pencils (in Russian). Trudy Sem. Petrovsk. 6 (1981), $97-146$.

[7] Mennicken, R. and Möller, M., Non-self-adjoint Boundary Eigenvalue Problems. North-Holland Mathematical Studies 192. Amsterdam: Elsevier 2003.

[8] Pivovarchik, V. and van der Mee, C., The inverse generalized Regge problem. Inverse problems 17 (2001), 1831 - 1845.

[9] Shubov, M., Asymptotic and spectral analysis of non-selfadjoint operators generated by a filament model with a critical value of a boundary parameter. Math. Methods Appl. Sci. 26 (2003)(3), 213 - 245.

[10] Whyburn, W. M., On self-adjoint ordinary differential equations of the fourth order. Amer. J. Math. 52 (1930)(1), $171-196$.

Received September 23, 2004; revised May 13, 2005 